\title{
BMJ Open Seroprevalence of anti-SARS-CoV-2 IgG among healthcare workers of a large university hospital in Milan, Lombardy, Italy: a cross-sectional study
}

Andrea Lombardi (D) , 1,2 Davide Mangioni, ${ }^{1}$ Dario Consonni, ${ }^{3}$ Lisa Cariani, ${ }^{4}$
Patrizia Bono, ${ }^{4}$ Anna Paola Cantù ${ }^{5}$ Basilio Tiso, ${ }^{5}$ Michele Carugno, ${ }^{3,6}$
Antonio Muscatello, ${ }^{1}$ Giovanna Lunghi, ${ }^{4}$ Angela Cecilia Pesatori, ${ }^{3,6}$
Luciano Riboldi, ${ }^{7}$ Ferruccio Ceriotti, ${ }^{4}$ Alessandra Bandera, ${ }^{1,2,8}$ Andrea Gori ${ }^{1,2,8}$

To cite: Lombardi A, Mangioni $D$, Consonni $D$, et al. Seroprevalence of anti-SARS-CoV-2 IgG among healthcare workers of a large university hospital in Milan, Lombardy, Italy: a crosssectional study. BMJ Open 2021;11:e047216. doi:10.1136/ bmjopen-2020-047216

- Prepublication history for this paper is available online. To view these files, please visit the journal online (http://dx.doi. org/10.1136/bmjopen-2020047216).

GL sadly passed away during the revision of this study.

AL and DM are joint first authors.

Received 21 November 2020 Revised 30 January 2021 Accepted 04 February 2021

Check for updates

(C) Author(s) (or their employer(s)) 2021. Re-use permitted under CC BY-NC. No commercial re-use. See rights and permissions. Published by BMJ.

For numbered affiliations see end of article.

Correspondence to

Dr Andrea Lombardi;

andrealombi89@gmail.com

\section{ABSTRACT}

Objectives To assess the seroprevalence of anti-SARSCoV-2 IgG among health careworkers (HCWs) in our university hospital and verify the risk of acquiring the infection according to work area.

Design Cross-sectional study.

Setting Monocentric, Italian, third-level university hospital.

Participants All the employees of the hospital on a voluntary base, for a total of 4055 participants among 4572 HCWs (88.7\%).

Primary and secondary outcome measures Number of anti-SARS-CoV-2 positive serology according to working area. Association of anti-SARS-CoV-2 positive serology to selected variables (age, gender, country of origin, body mass index, smoking, symptoms and contact with confirmed cases).

Results From 27 April 2020 to 12 June 2020, 4055 HCWs were tested and $309(7.6 \%)$ had a serological positive test. No relevant difference was found between men and women ( $8.3 \%$ vs $7.3 \%, p=0.3$ ), whereas a higher prevalence was observed among foreign-born workers $(27 / 186,14.5 \%, \mathrm{p}<0.001)$, employees younger than $30(64 / 668,9.6 \%, \mathrm{p}=0.02)$ or older than 60 years $(38 / 383,9.9 \%, p=0.02)$ and among healthcare assistants $(40 / 320,12.5 \%, p=0.06)$. Working as frontline HCWs was not associated with an increased frequency of positive serology $(p=0.42)$. A positive association was found with presence and number of symptoms $(p<0.001)$. The symptoms most frequently associated with a positive serology were taste and smell alterations (OR 4.62, $95 \% \mathrm{Cl}: 2.99$ to 7.15 ) and fever (OR 4.37, 95\% Cl: 3.11 to 6.13). No symptoms were reported in $84 / 309$ (27.2\%) HCWs with positive IgG levels. Declared exposure to a suspected/confirmed case was more frequently associated $(p<0.001)$ with positive serology when the contact was a family member $(19 / 94,20.2 \%)$ than a patient or colleague (78/888, 8.8\%).

Conclusions SARS-CoV-2 infection occurred undetected in a large fraction of HCWs and it was not associated with working in COVID-19 frontline areas. Beyond the hospital setting, exposure within the community represents an additional source of infection for HCWs.

\section{Strengths and limitations of this study}

- The serological test employed in our study has, after $>15$ days from the infection, a declared sensitivity of $97.4 \%$ and a specificity of $98.5 \%$.

- We performed our study on a large cohort of healthcare workers, from an area with a high incidence of COVID-19.

- Our study was monocentric and performed in Italy, therefore the results may be applicable only to similar scenarios (eg, Western countries with public health system).

\section{INTRODUCTION}

As of January 2021, the ongoing pandemic of coronavirus disease 2019 (COVID-19) caused by the severe acute respiratory syndrome coronavirus 2 (SARS-CoV-2) has affected more than 100 million people worldwide resulting in more than 2 million deaths. ${ }^{1}$ Since the beginning of the pandemic, healthcare workers (HCWs) have been identified as a group at high risk of infection. ${ }^{2}$ The occurrence of nosocomial transmission of SARS-CoV-2 has been well described, emphasising the adherence to infection control measures among HCWs to protect themselves and avoid nosocomial outbreaks. ${ }^{2-5}$ Conversely, other studies did not find differences in SARS-CoV-2 infection rates between frontline and non-frontline HCWs and between HCWs and the general population, suggesting community over nosocomial acquisition as major source of infection. ${ }^{6-8}$

In the current pandemic scenario, the optimal method to screen HCWs is still under debate. At present, the most frequently employed testing strategy is the detection of SARS-CoV-2 RNA through reverse transcriptase PCR on upper respiratory specimens 
in symptomatic individuals or in those exposed to confirmed cases of COVID-19. Unfortunately, the testing strategy based solely on upper respiratory specimens has significant limitations. In a large meta-analysis, the rate of positive nasopharyngeal swabs (NPS) ranged from 25\% to $80 \%$ and decreased with time and in asymptomatic or pauci-symptomatic cases. ${ }^{9}$ Of note, no data on test sensitivity in asymptomatic infected individuals exists, and clinical symptoms of COVID-19 among infected HCWs are often relatively mild, with fever and dyspnoea reported in $38 \%$ to $60 \%$ and $13 \%$ to $47 \%$ of cases, respectively. ${ }^{237810}$ It is also not uncommon for HCWs to work with mild symptoms, ${ }^{811}$ which increases the hazard of nosocomial outbreaks.

More recently, the serological assessment of SARS-CoV-2 infection has been proposed as screening strategy among both HCWs and the general population. Antibody sensitivity is $30 \% 1$ week after symptoms onset and rises to $70 \%$ and $>90 \%$ at 2 and 3 weeks, respectively. ${ }^{12}$ Hence, the most useful role for serology consists in detecting previous SARS-CoV-2 infection as screening strategy in exposed or high-risk HCWs. Little is known about the duration of humoral immune response to SARS-CoV-2 infection. In some studies antibody titres did not decline within 6 months after diagnosis. ${ }^{13-15}$ Conversely, others have reported a rapid waning over 3 to 4 months. ${ }^{1617}$

Here we present the results of SARS-CoV-2 serology assessment performed on HCWs from 27 April 2020 to 12 June 2020 at the Fondazione IRCCS Ca' Granda Ospedale Maggiore Policlinico located in Milan, Lombardy, by far the Italian region mostly affected by COVID-19. To cope with the COVID-19 emergency, the organisation of our hospital has been modified, and different wards have been entirely dedicated to the management of patients with COVID-19 to accommodate 350 of them. ${ }^{18}$ We evaluated the association between positive tests and demographic characteristics, occupation and working environment (frontline vs non-frontline HCWs). In addition, we assessed the frequency of positive tests in HCWs with previous symptoms of COVID-19 or who had been quarantined or in contact with suspected or proven COVID-19 cases.

\section{METHODS}

We collected occupational and clinical characteristics of all the consecutive HCWs who performed a serological assay for SARS-CoV-2 at the Fondazione IRCCS Ca' Granda Ospedale Maggiore Policlinico in Milan, Italy, from 27 April 2020 to 12June 2020. Of note, the first documented case of COVID-19 in our hospital occurred on 23 February 2020. Policlinico Hospital is one of the leading Italian hospitals in clinical and research activities located in Milan, northern Italy, with more than 4750 HCWs, 900 beds and 36000 hospitalisations per year. From 21 February 2020, to cope with the COVID-19 emergency, the hospital organisation was quickly modified with the installation of four different pavilions entirely dedicated to the management of patients with COVID-19 to accommodate 350 patients, of which 50 in intensive care units. Specific clinical pathways for patients with COVID-19 were created for critical settings (ie, triage and emergency ward, operating rooms, radiology department) and several internal guidelines were implemented and periodically updated. Trainings on donning and doffing of personal protective equipment (PPE) were provided by the infectious disease specialists and anaesthesiologists to the HCWs working in COVID-19 areas. Trainings were targeted to physicians, nurses and health assistants and consisted in brief reviews on COVID-19 clinical and epidemiological issues, set-up of COVID-19 wards in contaminated, buffer and clean areas, guidance on proper use of PPE in patient daily care and in specific situations (ie, patient transportation, dialysis, surgical interventions including childbirth).

The serological assay was offered freely to all hospital HCWs. At blood drawing, HCWs were asked to complete a questionnaire containing demographics, occupational and clinical characteristics. Information on age, gender, nationality, body mass index (BMI), smoking and comorbidities (hypertension, diabetes, immunosuppressive therapies, cardiac, respiratory or renal chronic diseases) was registered. HCWs were stratified by working environment in frontline and non-frontline workers (whether they provided direct assistance to patients with COVID-19 or not) and by job title in physicians (including residents), nurses and midwives, healthcare assistants, health technicians and clerical workers and technicians. The presence of any of the following symptoms since the end of February 2020 was collected: fever, cough, dyspnoea, diarrhoea, nausea or vomit, ageusia/dysgeusia or anosmia/parosmia, rhinorrhoea, ocular symptoms, sore throat, headache, myalgia and asthenia. The presence of any of the following indicators of previous exposure to SARS-CoV-2 was investigated: previous NPS (date and results), prophylaxis for SARS-CoV-2 infection (day and type of medication), home quarantine (period) and contact with suspected or proven COVID-19 cases (date and type of exposure).

The study was conducted in accordance with the Helsinki Declaration.

\section{SARS-CoV-2 serology}

SARS-CoV-2 serology was performed with LIAISON SARS-CoV-2 S1/S2 IgG test on LIAISON XL (DiaSorin, Saluggia, Italy). The test is a chemiluminescent immunoassay that detects quantitative anti-S1 and anti-S2 specific IgG antibodies against SARS-CoV-2 in human serum. The test has, after $>15$ days from the infection, a declared sensitivity of $97.4 \%$, and a specificity of $98.5 \%$. A test was considered positive when the value observed was equal to or above $15 \mathrm{AU} / \mathrm{mL}^{19}$

\section{Statistical analysis}

We calculated the adjusted seroprevalence using the formula: adjusted prevalence $=$ (observed 
prevalence + specificity-1) / (sensitivity+specificity-1), ${ }^{20}$ where sensitivity and specificity were those declared by the manufacturer.

We compared the prevalence of positive tests according to selected variables using $\chi^{2}$ tests. We then calculated ORs and $95 \%$ CIs by fitting a multivariable logistic regression model containing the following covariates: country of origin, gender, age class, occupation, frontline work, BMI class and cigarette smoking. For other variables (quarantine, symptoms, contact with COVID-19 case, prophylaxis/therapy and NPS), we used univariate logistic models. We evaluated the discriminating ability of the number of reported symptoms in a multivariable logistic regression model containing all groups of symptoms. Area under the ROC curve (AUC) was calculated after these models. To verify possible changes in IgG positivity over time, among HCWs with a previous positive NPS, we analysed the percentage of subjects with elevated IgG levels according to the days elapsed since the first positive NPS using logistic regression. Statistical analysis was performed with Stata 16 (StataCorp, 2019).

\section{Patient and public involvement}

The serologic assessment was freely offered to all the healthcare workers of our hospital. The majority of them (4055/4572, 88.7\%) participated and autonomously completed a questionnaire.

\section{RESULTS}

From 27 April 2020 to 12 June 2020, 4055 HCWs with a mean age of 44.8 years, 2823 women $(69.6 \%)$ and 1232 men $(30.4 \%)$, provided a blood sample and completed the questionnaire. The majority were physicians/ residents $(1292 / 4055,31.9 \%)$ and nurses/midwives $(1230 / 4055,30.3 \%)$. The overall frequency of workers with a positive test was $309 / 4055$ (7.6\%; $95 \%$ CI: $6.8 \%$ to $8.5 \%$ ) (table 1). The prevalence adjusted for declared test sensitivity and specificity would be $6.4 \%$. The frequency of positive tests was almost double among workers from abroad $(14.5 \%)$ compared with those of Italian ancestry $(7.3 \%)$, whereas women and men had a similar prevalence. The highest frequencies of a positive test were observed in the lowest $(<30$ years $)$ and highest $(\geq 60$ years) age classes. Across HCWs' job titles, a significant higher prevalence was detected among healthcare assistants $(40 / 320,12.5 \%)$, while weak differences were found for the other occupations $(6.0 \%$ to $8.0 \%)$. No difference was observed between frontline and non-frontline HCWs $(7.2 \%$ vs $7.9 \%)$. There was a positive trend of test positivity according to BMI, while current smokers had less than half the prevalence of test positivity than former and never smokers $(4.0 \%, 8.9 \%$ and $8.5 \%$, respectively). No association was found between test results and comorbidities (hypertension, diabetes, cardiac, respiratory or renal chronic diseases) or being on immunosuppressive treatment (data not shown). All findings of the univariate analyses were confirmed in the multivariable analysis.
Serology results stratified according to risk factors for previous exposure to SARS-CoV-2 are reported in table 2. A significant higher seropositivity was found among HCWs who had been quarantined $(166 / 426=39.0 \%$, OR=15.6 95\% CI: 12.0 to 20.1), who had taken antiviral drugs as treatment or prophylaxis $(44 / 135=32.3 \%$, $\mathrm{OR}=6.59,95 \% \mathrm{CI}: 4.51$ to 9.65$)$ and who had reported any symptom of SARS-Cov-2 infection in the preceding 4 weeks $(225 / 1511=14.9 \%, \mathrm{OR}=5.12,95 \%$ CI: 3.95 to 6.64$)$. We observed a clear monotonic increasing trend in test positivity with number of symptoms, from 56/608 (9.2\%) among HCWs with just one symptom to $62 / 170$ (36.5\%) in those with five or more. Conversely, no symptom was reported in 84/309 HCWs with positive serological test $(27.2 \%)$. The prevalence of positive tests was $5.6 \%$ (134/2372) in HCWs who did not report contacts with a person with COVID-19 and $10.1 \%(154 / 1525)$ in those who reported contacts with suspected or confirmed cases. Of note, prevalence of IgG positivity more than doubled if the reported contact was a family member $(19 / 94=20.2 \%)$ compared with a patient or a colleague $(78 / 888=8.8 \%)$. HCWs who had undergone SARS-CoV-2 NPS with negative result had a frequency of positive serology of $7.4 \%$ $(175 / 2375)$, almost the same as the overall hospital seroprevalence. On the contrary, the percentage of IgG positivity was much higher $(74.7 \%, 130 / 174)$ in those who had a positive NPS. In 162 subjects NPS had been performed before serology, while in 12 HCWs NPS was performed after the detection of a positive serology. Only four workers among the 1506 who had never performed NPS $(0.3 \%)$ had elevated IgG levels.

There were 162 subjects with a positive NPS before IgG testing. Among these, IgG testing was performed between 17 and 94 days (figure 1, left panel), with a peak between 49 and 63 days; the majority $(159,96.1 \%)$ were tested at least 21 days since the first positive swab. The percentage of positive IgG tests $(n=121)$ increased linearly (in the logit scale) over time (figure 1, right panel); it was $50 \%$ to $60 \%$ between 17 and 28 days, reaching $80 \%$ only after 60 days since the first positive NPS.

For every specific symptom, there was a positive association with elevated IgG levels (table 3). Specifically, strong associations emerged with fever $(19 / 374=31.8 \%)$ and with taste or smell alterations $(64 / 140=45.7 \%)$. In a multivariable model, these two symptoms were confirmed as the strongest predictors of positive test (both ORs $>4$ ). Other symptoms associated with positive SARS-CoV-2 serology were asthenia $(\mathrm{OR}=2.67)$, coryza $(\mathrm{OR}=1.90)$ and cough $(\mathrm{OR}=1.65)$, while sore throat was negatively associated with test positivity $(\mathrm{OR}=0.57)$. The AUC from the model containing all symptoms was 0.74 (95\% CI: 0.74 to $0.81)$.

\section{DISCUSSION}

In this study of HCWs of a large university hospital located in an area deeply affected by the COVID-19 pandemic, in a period ranging from 2 to 4 months after the first reported 
Table 1 Association between selected variables and prevalence of positive tests (anti-SARS-CoV-2 lgG $\geq 15$ AU/mL) among healthcare workers in a large university hospital, Milan, Italy, 27 April 2020 to 12 June 2020

\begin{tabular}{|c|c|c|c|c|c|c|}
\hline Variable & Workers & Positive test & & & & \\
\hline & $\mathbf{N}$ & $\mathbf{N}$ & $\%$ & P value $^{*}$ & ORt & $95 \% \mathrm{Cl} \dagger$ \\
\hline All & 4055 & 309 & 7.6 & & & \\
\hline \multicolumn{7}{|l|}{ Country of origin } \\
\hline Italy & 3869 & 282 & 7.3 & $<0.001$ & 1.00 & Reference \\
\hline Other & 186 & 27 & 14.5 & & 1.82 & 1.07 to 3.06 \\
\hline \multicolumn{7}{|l|}{ Gender } \\
\hline Women & 2823 & 207 & 7.3 & 0.30 & 1.00 & Reference \\
\hline Men & 1232 & 102 & 8.3 & & 1.13 & 0.85 to 1.52 \\
\hline \multicolumn{7}{|l|}{ Age (years) } \\
\hline$<30$ & 668 & 64 & 9.6 & 0.02 & 1.00 & Reference \\
\hline 30-39 & 1018 & 78 & 7.7 & & 0.74 & 0.51 to 1.07 \\
\hline $40-49$ & 858 & 48 & 5.6 & & 0.46 & 0.30 to 0.72 \\
\hline $50-59$ & 1128 & 81 & 7.2 & & 0.64 & 0.43 to 0.95 \\
\hline $60+$ & 383 & 38 & 9.9 & & 0.83 & 0.50 to 1.36 \\
\hline \multicolumn{7}{|l|}{ Occupation } \\
\hline Physicians, including residents & 1292 & 93 & 7.2 & 0.006 & 0.99 & 0.64 to 1.53 \\
\hline Nurses, midwives & 1230 & 99 & 8.0 & & 1.31 & 0.85 to 2.04 \\
\hline Healthcare assistants & 320 & 40 & 12.5 & & 1.84 & 1.04 to 3.25 \\
\hline Health technicians $\ddagger$ & 585 & 35 & 6.0 & & 0.84 & 0.50 to 1.40 \\
\hline Clerical workers, technicians & 628 & 42 & 6.7 & & 1.00 & Reference \\
\hline \multicolumn{7}{|l|}{ Frontline healthcareworkers } \\
\hline Never & 2061 & 149 & 7.2 & 0.42 & 1.00 & Reference \\
\hline Ever & 1730 & 137 & 7.9 & & 0.92 & 0.69 to 1.24 \\
\hline Missing & 264 & 23 & 8.7 & & & \\
\hline \multicolumn{7}{|l|}{$\mathrm{BMI}$} \\
\hline$<20$ & 684 & 46 & 6.7 & 0.04 & 0.90 & 0.62 to 1.32 \\
\hline 20-24.99 & 2035 & 145 & 7.1 & & 1.00 & Reference \\
\hline 25-29.99 & 945 & 79 & 8.4 & & 1.10 & 0.80 to 1.52 \\
\hline $30+$ & 314 & 31 & 9.9 & & 1.52 & 0.98 to 2.35 \\
\hline Missing & 77 & 8 & 10.4 & & & \\
\hline \multicolumn{7}{|l|}{ Cigarette smoking } \\
\hline Never & 2493 & 210 & 8.4 & $<0.001$ & 1.00 & Reference \\
\hline Former & 552 & 49 & 8.9 & & 1.12 & 0.79 to 1.58 \\
\hline Current & 842 & 34 & 4.0 & & 0.41 & 0.27 to 0.61 \\
\hline Missing & 168 & 16 & 9.5 & & & \\
\hline
\end{tabular}

${ }^{*}$ From $\chi^{2}$ test. For BMI: from $\chi^{2}$ test for trend. Missing data not included in analyses.

†From a multivariable logistic regression model including country of origin, gender, age, occupation, frontline area, BMI and smoking. Missing data not included in analyses.

‡Includes biologists, radiology and laboratory technicians, psychologists and other health technicians.

BMI, body mass index.;

case in the hospital, a relevant fraction of the personnel $(7.6 \%)$ showed anti-SARS-CoV-2 IgG values compatible with a previous infection. The highest rates of seroprevalence were detected among foreign-born workers, those belonging to extreme age groups (below 30 years and above 60 years) and healthcare assistants. SARS-CoV-2 seroprevalence of frontline HCWs did not differ from those who did not report direct contact with patients with COVID-19. Unsurprisingly, a large proportion (84/309, $27.2 \%$ ) of workers with a positive serology did not report 
Table 2 Association between quarantine, symptoms contact with patients with COVID-19, and prophylaxis and prevalence of positive tests (anti-SARS-CoV-2 lgG $\geq 15 \mathrm{AU} / \mathrm{mL}$ ) among healthcare workers in a large university hospital, Milan, Italy, 27 April 2020 to 12 June 2020

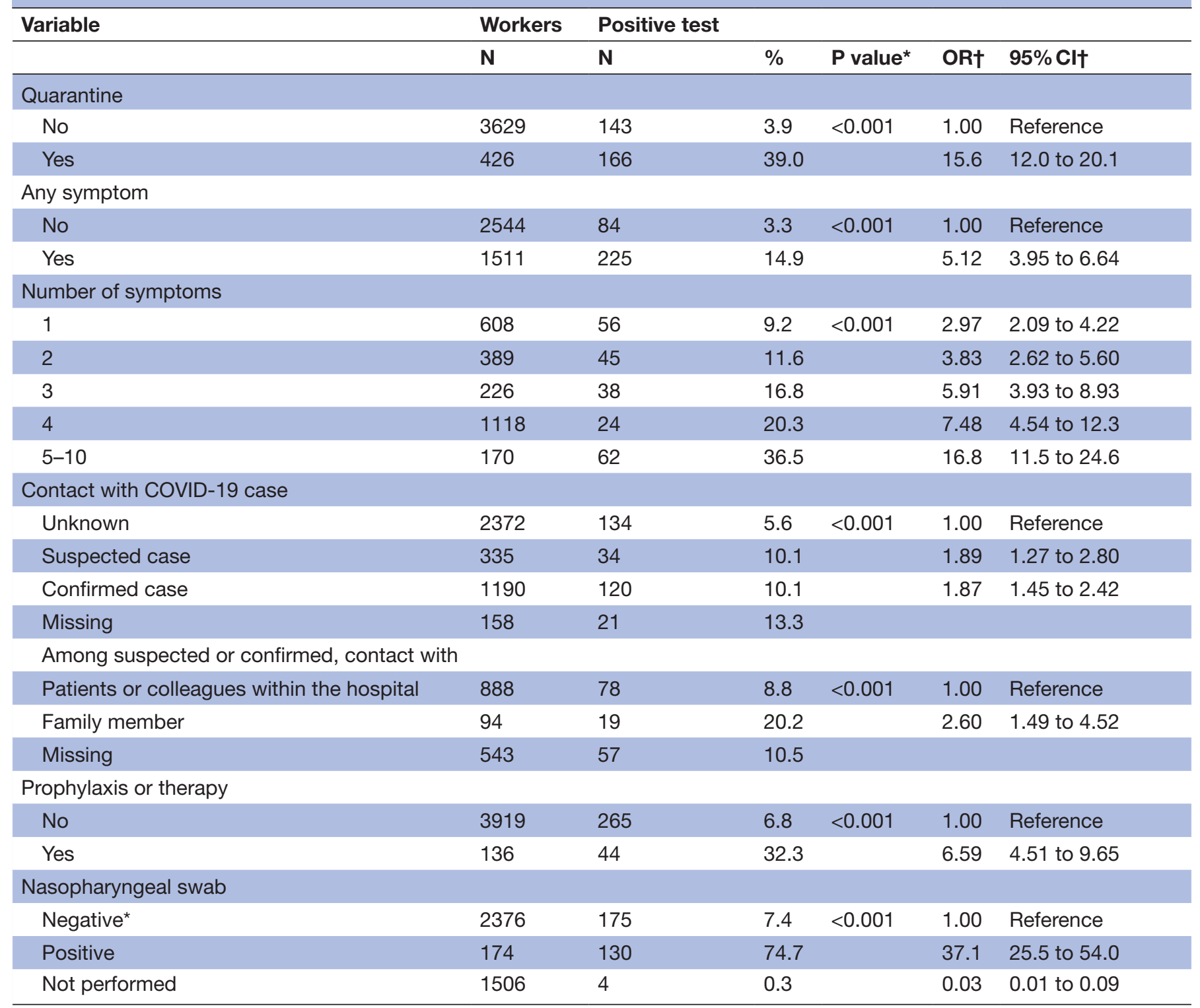

${ }^{*}$ From $\chi^{2}$ test. For number of symptoms: from $\chi^{2}$ test for trend. Missing data not included in analysis.

†From univariate logistic regression models. Missing data not included in analyses.

any symptom in the previous 4 weeks. Yet, HCWs who presented symptoms before the test, were quarantined, or took antiviral drugs as treatment or prophylaxis displayed higher positivity rates compared with those who did not. Interestingly, smokers had a significantly lower prevalence of positive serologies compared with non-smokers and former smokers. Finally, among symptoms, fever and smell and taste alteration were those more frequently associated with IgG positivity.

Our results are in accordance with the data presented by Sandri and colleagues, who described a rate of positive SARS-CoV-2 serologies (in their study defined as IgG >12 $\mathrm{AU} / \mathrm{mL}$ ) ranging from $6.4 \%$ to $9 \%$ among the HCWs of three different hospitals in Milan. ${ }^{21}$ In the same study the authors described a higher seroprevalence, between $35 \%$ and $43 \%$, in HCWs from Bergamo district, one of the areas in northern Italy most affected by COVID-19. These results are corroborated by the data provided by the Bergamo Health Authority, which reported a SARS-CoV-2 seroprevalence of $30.6 \%$ among HCWs from the Bergamo metropolitan area. Noteworthy is thus the fact that seroprevalence among HCWs mirrors the levels encountered in the general population, ranging from $7.1 \%$ and $56.9 \%$ in the Milan and Bergamo metropolitan area, respectively. ${ }^{22} 23$ Wide variations in seroprevalence among HCWs are reported worldwide, reflecting the distinct epidemiological scenarios occurring in each Country: SARS-CoV-2 seroprevalence of $1.6 \%, 3.8 \%, 5.0 \%, 9.3 \%, 19.1 \%, 24.4 \%$ 

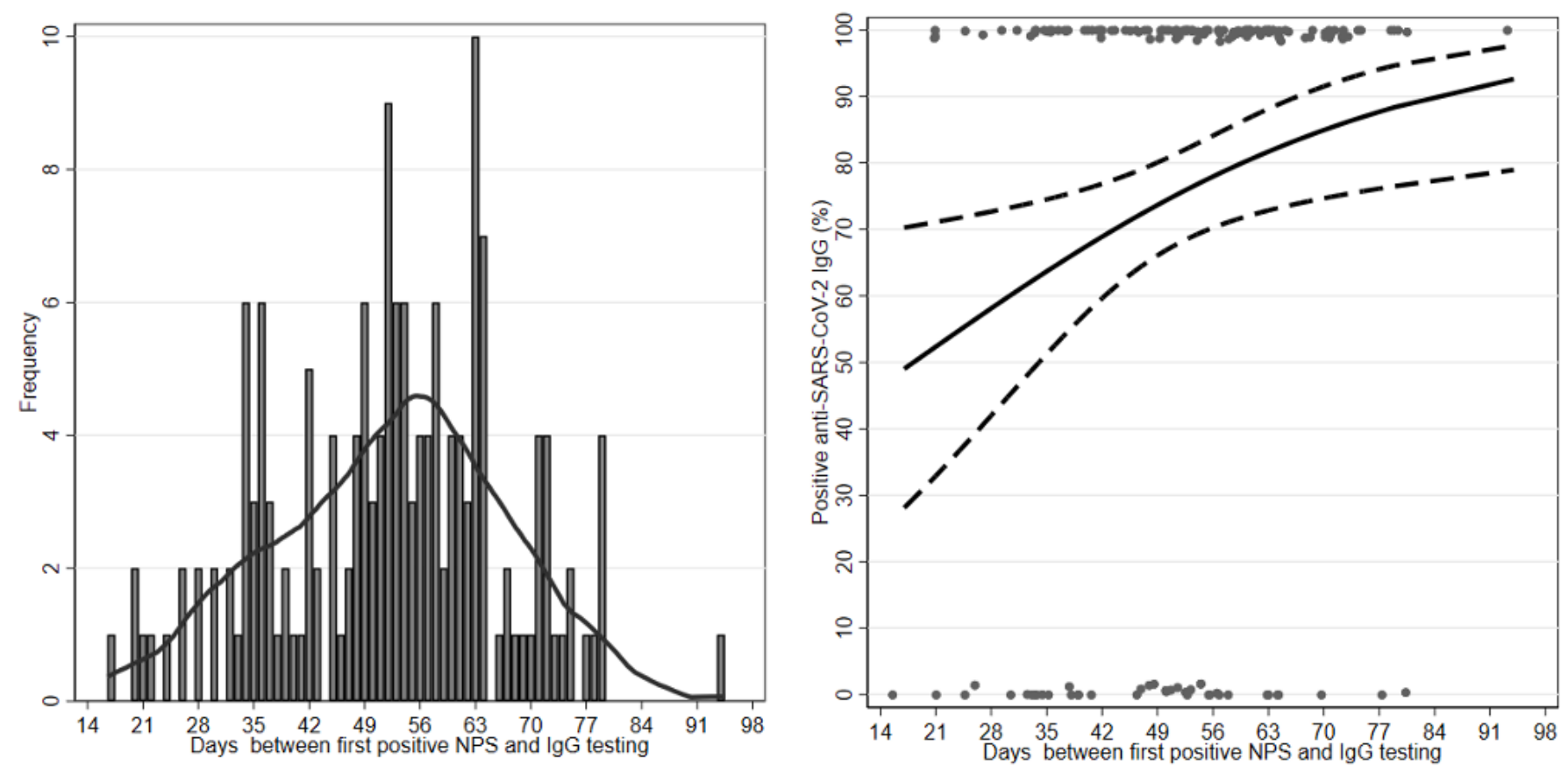

Figure 1 Number of IgG tests (left panel) and percentage of positive IgG tests (right panel) in 162 subjects with a positive nasopharyngeal swab (NPS) prior to serological testing, according to days elapsed since day of first positive NPS. Left panel shows histogram and kernel density smoothing line. In right panel circles indicate subjects with negative (lower circles, $n=41$ ) or positive (upper circles, $n=121$ ) anti-SARS-CoV-2 IgG, solid and dashed lines are the predicted percentages calculated with a logistic regression model, and dashed lines are $95 \%$ bands around the predicted.

and $33 \%$ are reported from studies conducted among HCWs in Germany, China, Netherlands, Spain, Sweden, UK and the USA, respectively. ${ }^{624-29}$

Contrasting findings exist regarding the role of direct assistance to patients with COVID-19 on the risk of SARS-CoV-2 infections in HCWs. Comparing frontline to non-frontline workers, we observed no difference in seroprevalence rates, in line with the findings of Mani and colleagues. ${ }^{7}$ At the same time, we observed a significantly higher seroprevalence among healthcare assistants $(40 / 320,12.5 \%)$, with all the other occupations (physician, nurses and midwives, technicians) below $8 \%$. A similar seroprevalence $(11.8 \%)$ was observed among healthcare assistants during the SARS pandemic in $2004 .{ }^{30}$ These results may suggest that, when nosocomial transmission occurs, it mainly involves those workers who have the closest contact with patients (eg, healthcare assistants who take care of patients' primary needs) and might therefore be at the highest risk. This condition may also reflect on the higher seroprevalence detected among HCWs from abroad. Indeed, a large fraction of this group is composed by healthcare assistants (46\%). When looking at healthcare assistants only, seroprevalence in workers from abroad was twice as high (20\%) than in workers of Italian ancestry $(9.8 \%)$.

What appears from our results is that SARS-CoV-2 transmission largely occurred from close contacts within the hospital in absolute terms (78 HCWs had contact with patients or colleagues, against 19 at home). However, in relative terms the prevalence was higher outside the hospital: in fact, HCWs who reported contacts with suspected or confirmed COVID-19 cases within the family had a prevalence of high IgG more than twice that of workers whose contacts were patients or colleagues (20.2\% vs $8.8 \%$, respectively). Similar results of family contacts as likely source of infection were reported by Sandri et al with even higher percentages $(31.2 \%)^{21}$ and were further corroborated by the molecular analyses performed by Sikkema $e t a l .{ }^{6}$

Regarding the lower prevalence of positive serologies among smokers, a protective effect of smoking on the risk of infection is unlikely. The lower seroprevalence we observed among smokers might reflect the influence of smoking on major components of both innate and adaptive immune cells. ${ }^{31}$ Particularly, a decreased production of IgA, IgG and IgM has been observed in smokers if compared with non-smokers. ${ }^{32}$

In our study, the positivity rate of anti-SARS-CoV-2 S1/ S2 IgG in HCWs who had a positive NPS (130/174, 74.7\%) is sensibly lower than the values reported by the manufacturer, which reports a sensitivity of $90.7 \%$ and $97.9 \%$ at 5 to 15 and $>15$ days after infection, respectively. ${ }^{19}$ Of note, $53 / 162(32.7 \%)$ of the tested workers performed serology 2 or more months after first NPS positivity (figure 1, left panel), and it is currently unknown for how long antibodies persist following SARS-CoV-2 infection. While in some studies antibody titres did not decline within 6 months after diagnosis, ${ }^{13-15}$ others reported a 
Table 3 Association between selected symptoms and prevalence of positive tests (anti-SARS-CoV-2 lgG $\geq 15$ AU/mL) among healthcare workers in a large university hospital, Milan, Italy, 27 April 2020 to 12 June 2020

\begin{tabular}{|c|c|c|c|c|c|c|}
\hline & Workers & Positive test & & & & \\
\hline & $\mathbf{N}$ & $\mathbf{N}$ & $\%$ & P value ${ }^{*}$ & ORt & $95 \% \mathrm{Cl} \dagger$ \\
\hline \multicolumn{7}{|c|}{ Specific symptom } \\
\hline \multicolumn{7}{|c|}{ Cough } \\
\hline No & 3523 & 201 & 5.7 & $<0.001$ & 1.00 & Reference \\
\hline Yes & 532 & 108 & 20.3 & & 1.65 & 1.18 to 2.30 \\
\hline \multicolumn{7}{|l|}{ Fever } \\
\hline No & 3681 & 190 & 5.2 & $<0.001$ & 1.00 & Reference \\
\hline Yes & 374 & 119 & 31.8 & & 4.37 & 3.11 to 6.13 \\
\hline \multicolumn{7}{|c|}{ Sore throat } \\
\hline No & 3677 & 261 & 7.1 & $<0.001$ & 1.00 & Reference \\
\hline Yes & 378 & 48 & 12.7 & & 0.57 & 0.38 to 0.86 \\
\hline \multicolumn{7}{|c|}{ Coryza } \\
\hline No & 3882 & 268 & 6.9 & $<0.001$ & 1.00 & Reference \\
\hline Yes & 173 & 41 & 23.7 & & 1.90 & 1.21 to 2.98 \\
\hline \multicolumn{7}{|c|}{ Headache } \\
\hline No & 3920 & 277 & 7.1 & $<0.001$ & 1.00 & Reference \\
\hline Yes & 135 & 32 & 23.7 & & 0.96 & 0.58 to 1.61 \\
\hline \multicolumn{7}{|c|}{ Myalgias } \\
\hline No & 3423 & 216 & 6.3 & $<0.001$ & 1.00 & Reference \\
\hline Yes & 632 & 93 & 14.7 & & 0.77 & 0.54 to 1.11 \\
\hline \multicolumn{7}{|c|}{ Diarrhoea/nausea/vomit } \\
\hline No & 3633 & 254 & 7.0 & 0.006 & 1.00 & Reference \\
\hline Yes & 422 & 55 & 13.0 & & 0.85 & 0.58 to 1.24 \\
\hline \multicolumn{7}{|c|}{ Asthenia } \\
\hline No & 3619 & 199 & 5.5 & $<0.001$ & 1.00 & Reference \\
\hline Yes & 436 & 110 & 25.2 & & 2.67 & 1.87 to 3.80 \\
\hline \multicolumn{7}{|c|}{ Ocular symptoms } \\
\hline No & 3847 & 281 & 7.3 & 0.001 & 1.00 & Reference \\
\hline Yes & 208 & 28 & 13.5 & & 0.78 & 0.46 to 1.32 \\
\hline \multicolumn{7}{|c|}{ Dyspnoea } \\
\hline No & 3927 & 275 & 7.0 & $<0.001$ & 1.00 & Reference \\
\hline Yes & 128 & 34 & 26.6 & & 1.38 & 0.82 to 2.32 \\
\hline \multicolumn{7}{|c|}{ Taste and smell alterations } \\
\hline No & 3915 & 245 & 6.3 & $<0.001$ & 1.00 & Reference \\
\hline Yes & 140 & 64 & 45.7 & & 4.62 & 2.99 to 7.15 \\
\hline
\end{tabular}

${ }^{*}$ From $\chi^{2}$ test.

†From a multivariable logistic model including all symptoms.

rapid waning over 3 to 4 months. ${ }^{16}{ }^{17}$ In our cohort the percentage of positive IgG tests increased monotonically over time (figure 1 , right panel), supporting the persistence of anti-SARS-CoV-2 S1/S2 IgG up to 3 months from NPS positivity. On the other hand, we found that $7.4 \%$ of workers with negative NPS $(175 / 2375)$ had IgG $>15 \mathrm{AU} / \mathrm{mL}$. Unfortunately, we are unable to ascertain what proportion is due to lack of NPS sensitivity and what arises from imperfect specificity of IgG test. In fact, our study was not designed to assess the accuracy of the serological test. Further reports of real-life data are therefore needed.

Finally, positive serology was associated with a recent history of typical symptoms of SARS-CoV-2 infection, especially taste and smell alterations and fever. These findings corroborate previous observations made by our group who 
identified taste and smell alterations and fever as the symptoms most frequently reported in HCWs with SARS-Cov-2 positivity on NPS. ${ }^{10}$ Other authors confirmed the same observations, suggesting that anosmia is the symptom which better characterises COVID-19. ${ }^{21}{ }^{26}$ Notably, a large fraction of HCWs with positive serology $(84 / 309,27.2 \%)$ did not report any symptom in the 4 weeks before the test. This finding is also well-described in COVID-19 epidemiology, where the rate of asymptomatic or pauci-symptomatic infected persons ranges from $1.6 \%$ to $56.5 \%$ depending on subject characteristics and on the analysed country. ${ }^{32}$ Unfortunately, in hospital settings the absence of symptoms makes it difficult to identify infected HCWs and hampers many strategies to control the infection.

The first limitation of our work has been noted above: this study was performed for health surveillance purposes and thus not designed to evaluate serological test performance (sensitivity and specificity). Second, some degree of recall bias, that is, under-reporting of mild symptoms which occurred many weeks before serological test, is a possibility. In this case, we may have overestimated the proportion of asymptomatic workers with elevated IgG. Yet, considering that the study started at the end of April 2020, and that the COVID-19 pandemic in Lombardy begun at the end of February, we probably missed only a small percentage of subjects with clinical manifestations. Third, the serological assessment was not mandatory and was therefore not performed on all HCWs. Nevertheless, considering that the hospital employees are 4572, our study has involved a large fraction of them (4055/4572, $88.7 \%$ ) and thus provides a fair description of SARS-CoV-2 exposure in HCWs of our hospital. Finally, we could not evaluate the serological status of all HCWs in a single day. As the epidemic was still ongoing, even though on a much smaller scale (the zenith of the infection was in March), we may have missed a few new infections.

What is suggested by our study, and by those similarly performed in the same area in the context of the ongoing pandemic, ${ }^{21}$ is that the observed seroprevalence rate reflects the spread of infection in the community served by the hospital. Assuming that PPE is provided and correctly employed by all HCWs, hospitals do not seem to act as an epicentre of the infection. In our study, healthcare assistants showed the highest seroprevalence rate. We do believe that education and training of all HCWs should be strongly supported. Periodic training of correct use of PPE and infection control procedures should be addressed not only to physicians and nurses but also to other healthcare professionals.

The fact that more than one quarter of SARS-CoV-2 infections occurred unnoticed supports the implementation of systematic testing strategies among HCWs without an ascertained history of infection. Unfortunately, the best testing strategy as well as the timing and setting in which these tests have the highest performance is still uncertain. Future studies should address these gaps of knowledge. As of now, we deem it is important to monitor periodically SARS-CoV-2 serology in HCWs to correlate the seroprevalence rates with those of general population and detect any discrepancy. This will allow to implement timely and effective infection control measures, thus preventing hospitals to become drivers of future COVID-19 outbreaks.

\section{Author affiliations}

${ }^{1}$ Infectious Diseases Unit, Foundation IRCCS Ca' Granda Ospedale Maggiore Policlinico, Milan, Italy

${ }^{2}$ Department of Pathophysiology and Transplantation, University of Milan, Milano, Italy

${ }^{3}$ Epidemiology Unit, Foundation IRCCS Ca' Granda Ospedale Maggiore Policlinico, Milan, Italy

${ }^{4}$ Clinical Laboratory, Foundation IRCCS Ca' Granda Ospedale Maggiore, Milan, Italy ${ }^{5}$ Medical Direction, Foundation IRCCS Ca' Granda 0spedale Maggiore Policlinico, Milan, Italy

${ }^{6}$ Department of Clinical Sciences and Community Health, University of Milano, Milan, Italy

${ }^{7}$ Occupational Health Unit, Foundation IRCCS Ca' Granda Ospedale Maggiore Policlinico, Milan, Italy

${ }^{8}$ Centre for Multidisciplinary Research in Health Science (MACH), University of Milan, Milan, Italy

Acknowledgements This work is dedicated to the memory of GL, who sadly passed away during the revision of this paper. We thank the personnel of SPIO (Servizio Prevenzione e Igiene Ospedaliera), S Adamoli, F De Palo, M Gatti, L Guerrieri, P Marazzi, B Marinelli, M Polonioli, S Pratò, E Radice and D Serra for their help in data collection.

Contributors AL, DM, DC, AB and AG conceived the study. LC, PB, APC, BT, MC, $\mathrm{GL}, \mathrm{ACP}, \mathrm{LR}, \mathrm{AM}$ and FC collected the data and performed the serological survey. DC performed the statistical analyses. AL, DM and DC wrote the first draft of the manuscript. All authors revised the final version of the manuscript.

Funding The authors have not declared a specific grant for this research from any funding agency in the public, commercial or not-for-profit sectors.

Competing interests None declared.

Patient and public involvement Patients and/or the public were not involved in the design, or conduct, or reporting, or dissemination plans of this research.

Patient consent for publication Not required.

Ethics approval The study was approved by the institutional review board (368_2020bis) of our hospital.

Provenance and peer review Not commissioned; externally peer reviewed.

Data availability statement Data are available upon reasonable request. We encourage those interested to contact the corresponding author.

Open access This is an open access article distributed in accordance with the Creative Commons Attribution Non Commercial (CC BY-NC 4.0) license, which permits others to distribute, remix, adapt, build upon this work non-commercially, and license their derivative works on different terms, provided the original work is properly cited, appropriate credit is given, any changes made indicated, and the use is non-commercial. See: http://creativecommons.org/licenses/by-nc/4.0/.

ORCID iD

Andrea Lombardi http://orcid.org/0000-0002-0383-9579

\section{REFERENCES}

1 Johns Hopkins Coronavirus Resource Center. COVID-19 Map Johns Hopkins Coronavirus Resource Center, 2020. Available: https://coronavirus.jhu.edu/map.html

2 Chou R, Dana T, Buckley DI, et al. Epidemiology of and risk factors for coronavirus infection in health care workers: a living rapid review. Ann Intern Med 2020;173:120-36.

3 Lai X, Wang M, Qin C, et al. Coronavirus disease 2019 (COVID-2019) infection among health care workers and implications for prevention measures in a tertiary hospital in Wuhan, China. JAMA Netw Open 2020;3:e209666.

4 Nguyen LH, Drew DA, Joshi AD, et al. Risk of COVID-19 among frontline healthcare workers and the general community: a 
prospective cohort study. medRxiv 2020. doi:10.1101/2020.04.29.20 084111. [Epub ahead of print: 25 May 2020]

5 Gómez-Ochoa SA, Franco OH, Rojas LZ, et al. COVID-19 in healthcare workers: a living systematic review and meta-analysis of prevalence, risk factors, clinical characteristics, and outcomes. Am J Epidemiol 2021;190:161-75.

6 Sikkema RS, Pas SD, Nieuwenhuijse DF, et al. COVID-19 in healthcare workers in three hospitals in the South of the Netherlands: a cross-sectional study. Lancet Infect Dis 2020;20:1273-80.

7 Mani NS, Budak JZ, Lan KF, et al. Prevalence of COVID-19 infection and outcomes among symptomatic healthcare workers in Seattle, Washington. Clin Infect Dis 2020. doi:10.1093/cid/ciaa761. [Epub ahead of print: 16 Jun 2020].

8 den BM, Buiting AGM, Pas SD. SARS-CoV-2 infection in 86 healthcare workers in two Dutch hospitals in March. medRxiv 2020:1-16.

9 Mohammadi A, Esmaeilzadeh E, Li Y, et al. SARS-CoV-2 detection in different respiratory sites: a systematic review and meta-analysis. EBioMedicine 2020;59:102903-20.

10 Lombardi A, Consonni D, Carugno M, et al. Characteristics of 1573 healthcare workers who underwent nasopharyngeal swab testing for SARS-CoV-2 in Milan, Lombardy, Italy. Clin Microbiol Infect 2020;26:1413.e9-1413.e13.

11 Tartari E, Saris K, Kenters N, et al. Not sick enough to worry? "Influenza-like" symptoms and work-related behavior among healthcare workers and other professionals: Results of a global survey. PLoS One 2020;15:e0232168-14.

12 Deeks JJ, Dinnes J, Takwoingi Y, et al. Antibody tests for identification of current and past infection with SARS-CoV-2. Cochrane Database Syst Rev 2020;6:CD013652.

13 Gudbjartsson DF, Norddahl GL, Melsted P, et al. Humoral immune response to SARS-CoV-2 in Iceland. $N$ Engl J Med 2020;383:1724-34.

14 Wajnberg A, Amanat F, Firpo A, et al. Robust neutralizing antibodies to SARS-CoV-2 infection persist for months. Science 2020;370:1227-30.

15 L'Huillier AG, Meyer B, Andrey DO. Antibody persistence in the first six months following SARS-CoV-2 infection among hospital workers: a prospective longitudinal study. Clin Microbiol Infect.

16 Ward H, Cooke G, Atchison C. Declining prevalence of antibody positivity to SARS-CoV-2: a community study of 365000 adults. medXriv 2020.

17 Seow J, Graham C, Merrick B, et al. Longitudinal observation and decline of neutralizing antibody responses in the three months following SARS-CoV-2 infection in humans. Nat Microbiol 2020;5:1598-607.
18 Bandera A, Aliberti S, Gualtierotti R, et al. COVID-19 network: the response of an Italian reference Institute to research challenges about a new pandemia. Clin Microbiol Infect 2020;26:1576-8.

20 Sempos CT, Tian L. Adjusting coronavirus prevalence estimates for laboratory test kit error. Am J Epidemiol 2021;190:109-15.

21 Sandri MT, Azzolini E, Torri V. IgG serology in health care and administrative staff populations from 7 hospitals representative of different exposures to SARS-CoV-2 in Lombardy, Italy. medXriv 2020.

22 Coronavirus: I dati dei test sierologici effettuati nella Bergamasca dal 23 aprile al 3 giugno, 2020. Available: https://www.ats-bg.it/upload/ asl_bergamo/gestionedocumentale/CSATSBG2020-06-08coronaviru sesitisierologici_784_31055.pdf

23 Valenti L, Bergna A, Pelusi S, et al. SARS-CoV-2 seroprevalence trends in healthy blood donors during the COVID-19 outbreak in Milan. Blood Transfus 2021. doi:10.2450/2021.0324-20. [Epub ahead of print: 03 Feb 2021].

24 Korth J, Wilde B, Dolff S, et al. SARS-CoV-2-specific antibody detection in healthcare workers in Germany with direct contact to COVID-19 patients. J Clin Virol 2020;128:104437.

$25 \mathrm{Xu}$ X, Sun J, Nie S, et al. Seroprevalence of immunoglobulin M and G antibodies against SARS-CoV-2 in China. Nat Med 2020;26:1193-5.

26 Garcia-Basteiro AL, Moncunill G, Tortajada M, et al. Seroprevalence of antibodies against SARS-CoV-2 among health care workers in a large Spanish reference Hospital. Nat Commun 2020;11:1-36.

27 Rudberg A-S, Havervall S, Månberg A, et al. SARS-CoV-2 exposure, symptoms and seroprevalence in healthcare workers in Sweden. Nat Commun 2020;11:1-8

28 Shields AM, Faustini SE, Perez-Toledo M. SARS-CoV-2 seroconversion in health care workers. medXriv 2020.

29 Mansour M, Leven E, Muellers K. Prevalence of SARS-CoV-2 antibodies among healthcare workers at a tertiary academic hospital in New York City. J Gen Intern Med 2020:2-3.

30 Ip M, Chan PKS, Lee N, et al. Seroprevalence of antibody to severe acute respiratory syndrome (SARS)-associated coronavirus among health care workers in SARS and non-SARS medical wards. Clin Infect Dis 2004;38:e116-8.

31 Qiu F, Liang C-L, Liu H, et al. Impacts of cigarette smoking on immune responsiveness: up and down or upside down? Oncotarget 2017;8:268-84

32 Giuca M, Pasini M, Tecco S, et al. Levels of salivary immunoglobulins and periodontal evaluation in smoking patients. BMC Immunol 2014;15:5-6.

33 Gao Z, Xu Y, Sun C, et al. A systematic review of asymptomatic infections with COVID-19. J Microbiol Immunol Infect 2020;323. 